\title{
Resistência de Cooperia spp. e Haemonchus spp. às avermectinas em bovinos de corte
}

[Resistance of Cooperia spp. and Haemonchus spp. to avermectins in beef cattle]

\author{
V.B. Rangel ${ }^{1}$, R.C. Leite $^{2}$, P.R. Oliveira ${ }^{2}$, E.J. Santos $J r^{1}$ \\ ${ }^{1}$ Akzo Nobel Ltda - Divisão Intervet \\ Av. Alfredo Egídio de S. Aranha, 100 \\ 07426-170 - São Paulo, SP \\ ${ }^{2}$ Escola de Veterinária-UFMG
}

\begin{abstract}
RESUMO
Foram realizados dois testes de redução de ovos de helmintos por grama de fezes em bovinos naturalmente infectados. No primeiro teste utilizaram-se quatro grupos tratados: ivermectina 1\% (IVM), produto endectocida experimental (PEE), ivermectina LA 1\% (IVMLA) e doramectina 1\% (DRM). A contagem de ovos por gramas de fezes (OPG) foi realizada durante 14 dias pós-tratamento. Foram observadas as seguintes taxas de eficácia: IVM, -1,3\%; PEE, 100\%; IVMLA, 18,9\% e DRM 50,6\%. Os gêneros de helmintos encontrados foram Cooperia e Haemonchus. No segundo teste foram avaliadas moxidectina $1 \%$ e abamectina $1 \%$, e ambas apresentaram eficácia acima de $99 \%$.
\end{abstract}

Palavras-chave: helmintos gastrintestinais, avermectina, resistência, controle

\begin{abstract}
Two fecal count-reducing-tests in infected bovines were performed considering eggs per gram. In the first test it was used four treated groups with ivermectin 1\% (IVM), experimental endectocid product (EEP), ivermectin LA 1\% (IVMLA) e doramectin 1\% (DRM). Evaluating the finds of eggs per gram in a 14-dayperiod after treatment and comparing with the control group it was observed the following efficacy rates: $1.3 \%$ to IVM, 100.0\% to EEP, 18.9\% to IVMLA and 50.6\% to DRM. Helminthes genders found were Cooperia and Haemonchus. The second test was performed to evaluate moxidectin $1 \%$ and abamectin $1 \%$ and results showed efficacy over $99.0 \%$ for both.
\end{abstract}

Keywords: gastrointestinal helminthes, avermectin, control, helminthes resistance

\section{INTRODUÇÃO}

O controle dos helmintos tem sido realizado principalmente com produtos químicos. Estes produtos são largamente utilizados na pecuária de corte e, muitas vezes, administrados sem critérios epidemiológicos, permitindo o aparecimento de resistência. Se o uso for intensivo e o intervalo entre tratamentos se aproximar do período pré-patente dos nematódeos, os parasitos resistentes serão capazes de continuar ininterruptamente sua reprodução no hospedeiro, enquanto os espécimes sensíveis terão poucas oportunidades de infectar os animais, alcançar maturidade e produzir ovos antes de serem expostos ao próximo tratamento. Esse tipo de manejo, influenciando a resistência anti-helmíntica à ivermectina foi observado em ovinos por Veríssimo et al. (2002) e Echevarria et al. (1996). Taylor et al. (2002) afirmaram que o uso intensivo de anti-helmínticos para o controle de infecções desses parasitos resultou no

Recebido para publicação em 9 de junho de 2003

Recebido para publicação, após modificações, em 19 de abril de 2004

E-mail: vandersonrangel@yahoo.com.br 
desenvolvimento de resistência e se tornou o maior problema prático da criação de animais.

Este trabalho teve como objetivo verificar o grau de sensibilidade dos helmintos gastrintestinais de bovinos de corte a produtos a base de lactonas macrocíclicas

\section{MATERIAL E MÉTODOS}

O experimento foi conduzido em uma fazenda, localizada no município de Betim, região metalúrgica de Minas Gerais, latitude 1951'39', e longitude 44¹0'55', com altitude de 800 metros, clima ameno e precipitação pluviométrica anual média de $1.400 \mathrm{~mm}$. As estações de seca e chuvas são bem definidas, com mais de $90 \%$ das chuvas ocorrendo de outubro a março.

No laboratório de ectoparasitoses foram realizados os testes de contagem de ovos por grama de fezes (OPG) e as culturas de larvas ou coproculturas. As larvas foram identificadas no laboratório de helmintologia veterinária do Instituto de Ciências Biológicas da UFMG.

O calendário de vermifugação realizado na propriedade consistiu em aplicações de doramectina nos meses de janeiro, março, maio e novembro para os bezerros lactentes. Para os animais desmamados até 24 meses de idade, fezse o uso de avermectinas em janeiro, março, maio, julho, setembro e novembro. $\mathrm{O}$ uso de produtos à base de avermectinas vem ocorrendo a mais de cinco anos sem registros específicos de identificação da base química utilizada.

De um lote de 200 animais sem histórico de aplicação de parasiticidas há pelo menos 60 dias, foram selecionados 80 bovinos da raça Canchim, machos, desmamados e com infestação natural por parasitos. A idade variou de sete a nove meses e o peso de 206 e $298 \mathrm{~kg}$ de peso vivo.

Os animais foram manejados em pastos com predomínio de Brachiaria brizantha, a lotação média foi de três animais/ha e o sistema de pastejo foi o contínuo de carga variável. Água e sal mineral foram fornecidos ad libitum.
Foram realizados dois testes de redução de contagem de ovos por grama de fezes. Os testes foram realizados de acordo com as recomendações da World Association for the Advancement of Veterinary Parasitology (WAAP; Coles et al., 1992). Para efeito de análise estatística, amostras de fezes de animais com contagens inferiores a 150 OPG foram desprezadas. No $14^{\circ}$ dia após o tratamento (PT) foram novamente colhidas fezes dos animais e realizado novo exame de OPG.

No primeiro teste, iniciado em agosto de 2002, foram utilizados três grupos com 13 animais cada, utilizando diferentes produtos: T2, T3, T4 e um grupo-controle T1 com 11 animais. O segundo teste teve início em setembro de 2002 e foram utilizados um grupo controle T5, com 11 animais, e outros dois grupos utilizando diferentes produtos: T6 e T7, com 15 animais cada. No dia anterior ao início do experimento, os animais de ambos os testes foram identificados com os brincos numerados e classificados de acordo com a contagem de OPG.

No dia 0 de cada teste os animais de cada grupo controle, $\mathrm{T} 1$ e $\mathrm{T} 5$, receberam tratamento placebo (solução salina injetável) e serviram de controle negativo. No mesmo dia os animais dos outros grupos receberam os tratamentos com os produtos administrados em doses de acordo com o peso vivo.

No primeiro teste os animais do T2, T3 e T4 receberam produtos à base de ivermectina $1 \%$, ivermectina LA $1 \%$ e doramectina $1 \%$, respectivamente, administrados por via subcutânea na dose de $200 \mu \mathrm{g} / \mathrm{kg}$ de peso vivo. No segundo teste, os grupos T6 e T7 receberam produtos à base de moxidectina $1 \%$ e abamectina LA $1 \%$, respectivamente, administradas via subcutânea na dose de $200 \mu \mathrm{g} / \mathrm{kg}$ de peso vivo.

As fezes foram coletadas diretamente da ampola retal com saco plástico, acondicionadas em isopor com gelo e posteriormente em geladeira até a realização dos exames. Os exames de fezes foram realizados pelo método de Gordon e Whitlock (1939) modificado, com sensibilidade de detecção de 50 ovos por grama de fezes. Selecionadas as cinco amostras com maior 


\section{Rangel et al.}

contagem de ovos de cada grupo, elas foram homogeneizadas e a partir delas realizada a cultura de larvas pela técnica de Roberts e O’Sullivan (1949) modificada.

Para determinar a redução dos ovos por grama de fezes e a resistência foi utilizada a fórmula proposta por Coles et al. (1992):

$\mathrm{PR} \%=100(1-\mathrm{Xt} / \mathrm{Xc})$, em que

PR\% é o percentual de redução da contagem de ovos; Xt a média aritmética da contagem de ovos do grupo tratado no dia $14^{\circ}$ dia PT; e Xc a média aritmética da contagem de ovos do grupo controle no dia $14^{\circ}$ dia PT.

Seguindo as recomendações da WAAVP, os helmintos foram considerados resistentes quando a porcentagem de redução na contagem dos ovos foi menor do que $95 \%$ (Coles et al., 1992).

\section{RESULTADOS}

Em ambos os testes a contagem média de OPG inicial de todos os grupos estava elevada. No primeiro teste a taxa de redução na contagem de OPG dos grupos tratados foi inferior a $95 \%$. No segundo teste, foi acima de 95\% (Tab. 1).

No primeiro teste, na coprocultura após o tratamento, foram observadas larvas dos gêneros Haemonchus e Cooperia. Houve predominância de helmintos do gênero Haemonchus, 75\% e $72 \%$ nos grupos T2 e T3, respectivamente. Em T4 predominou o gênero Cooperia, $85 \%$ (Tab. 2).

Os gêneros presentes na coprocultura prétratamento, em todos os grupos do segundo teste e no T5 após o tratamento foram Cooperia e Haemonchus (Tab. 2).

Tabela 1. Contagem média de ovos por gramas de fezes (OPG), inicial e final, e taxa de redução de acordo com os tratamentos

\begin{tabular}{llccc}
\hline \multirow{2}{*}{ Tratamento } & \multicolumn{3}{c}{ OPG } & \multirow{2}{*}{ Redução (\%) } \\
\cline { 2 - 3 } & Inicial & Final & - \\
T1 & Controle & 1.371 & 1.200 & $-1,3$ \\
T2 & Ivermectina 1\% & 1.177 & 1.215 & 18,9 \\
T3 & Ivermectina LA 1\% & 1.233 & 973 & 50,6 \\
T4 & Doramectina 1\% & 1.046 & 592 & - \\
T5 & Controle & 985 & 631 & 100,0 \\
T6 & Moxidectina 1\% & 547 & 0 & 99,0 \\
T7 & Abamectina LA 1\% & 1.175 & 6 & \\
\hline
\end{tabular}

Tabela 2. Gênero de larvas de helmintos (\%) em coproculturas realizadas antes e após o tratamento de bovinos de corte naturalmente infectados

\begin{tabular}{|c|c|c|c|c|c|c|c|}
\hline \multirow{2}{*}{ Tratamento } & \multicolumn{4}{|c|}{ Inicial } & \multicolumn{3}{|c|}{ Final } \\
\hline & Coop & Haem & Oesop & Total & Coop & Haem & Total \\
\hline T1 Controle & 45 & 40 & 15 & 100 & 65 & 35 & 100 \\
\hline T2 Ivermectina $1 \%$ & 56 & 44 & 0 & 100 & 25 & 75 & 100 \\
\hline T3 Ivermectina LA $1 \%$ & 43 & 57 & 0 & 100 & 28 & 72 & 100 \\
\hline T4 Doramectina 1\% & 35 & 65 & 0 & 100 & 85 & 15 & 100 \\
\hline T5 Controle & 66 & 19 & 15 & 100 & 47 & 53 & 100 \\
\hline T6 Moxidectina $1 \%$ & 68 & 32 & 0 & 100 & 0 & 0 & 0 \\
\hline T7 Abamectina LA 1\% & 77 & 23 & 0 & 100 & 0 & 0 & 0 \\
\hline
\end{tabular}

Coop.: Cooperia; Haem.: Haemonchus; Oesop.: Oesophagostomum.

\section{DISCUSSÃO}

O aparecimento de marcas comerciais de ivermectina $1 \%$ associado ao baixo preço do produto resultou no uso intensivo e indiscriminado de endectocidas nos rebanhos bovinos. Isto tem provocado o aumento da resistência em helmintos.

Mottier e Lanusse (2001) conceituam resistência adquirida às drogas como o fenômeno que se dá quando populações inicialmente susceptíveis à 
ação de um fármaco o deixam de ser devido à ocorrência de modificações genéticas. Segundo Echevarria (2002), a resistência é um aumento no número de indivíduos capazes de suportar doses de um composto químico que tenha provado ser letal à maioria da população normalmente sensível.

O teste de redução de OPG é o mais utilizado no mundo para identificar helmintos resistentes. Contudo, é limitado, pois só mede a produção de ovos de fêmeas adultas e nem sempre há correlação entre essa característica e a carga de vermes. Alta correlação entre contagem de ovos nas fezes e contagem de vermes foi encontrada para Haemonchus sp, mas não para Trichostrongylus columbriformes ou Ostertagia circumcincta. A praticidade e a relativa acurácia faz com que esse teste possa ser realizado em larga escala (Taylor et al., 2002).

Com base na contagem de ovos de helmintos e nos resultados da coprocultura, há evidências de provável resistência da população de Cooperia e Haemonchus às bases doramectina e ivermectina, utilizando os critérios recomendados por Coles et al. (1992). Pelos mesmos critérios, observa-se que a população de helmintos foi sensível à moxidectina e à abamectina LA.

Dados semelhantes foram registrados na Argentina por Anziani et al. (2000), que ao utilizarem o mesmo método de detecção in vivo, encontraram helmintos do gênero Cooperia resistente à ivermectina $1 \%$, ivermectina $3,15 \%$, doramectina $1 \%$ e provavelmente à moxidectina. Ao utilizarem o teste de redução de contagem de ovos fecais (FECRT) e o teste controlado, Fiel et al. (2001) também encontraram helmintos do gênero Cooperia resistentes à ivermectina $1 \%$, ivermectina $1 \%$ LA e doramectina $1 \%$.

No Brasil, também foram relatados dados semelhantes de resistência de Cooperia punctata à ivermectina $1 \%$ e doramectina $1 \%$ por Cardoso et al.(2002) e resistência do gênero Haemonchus à ivermectina $1 \%$ por Souza et al. (2002). Todos esses relatos de resistência e os do presente trabalho sugerem algum mecanismo similar de desenvolvimento de resistência às duas bases químicas, ivermectina $1 \%$ e doramectina $1 \%$.

A maior prevalência de Haemonchus nos grupos tratados com ivermectina sugere baixa eficiência dessa base. No grupo tratado com doramectina, o problema maior parece ser com o gênero Cooperia, pois sua prevalência foi maior do que a do gênero Haemonchus.

A diferença no modo de ação da ivermectina e da moxidectina, descrita por Torrano (2003), explica a eficácia da segunda sobre a primeira. O resultado observado para abamectina 1\%, semelhante ao relatado por Cardoso (2001), permite afirmar que essa base também é diferente da ivermectina quanto ao desenvolvimento da resistência. Estes resultados demonstram provável resistência à ivermectina $1 \%$ e doramectina $1 \%$ nessa população de helmintos dos gêneros Cooperia e Haemonchus, e sugerem a moxidectina $1 \%$ e a abamectina $1 \%$ como opções para o controle dessa população.

\section{REFERÊNCIAS BIBLIOGRÁFICAS}

ANZIANI, O.S.; ZIMMERMANN, G.; GUGLIELMONE, A.A. et al. Resistance to avermectinas of parasitized bovines by Cooperia spp. Vet. Arg., v.17, p.280-281, 2000.

CARDOSO, J.M.S. Identificação de Cooperia punctata (LINSTOW, 1907) resistente a ivermectin e doramectin em bovinos no estado do Rio de Janeiro, Basil. 2001. Dissertação (Mestrado em Medicina Veterinária) Universidade Federal Rural do Rio de Janeiro.

CARDOSO, J.M.S; SANT'ANA, F.B.; MARTINS, I.V.F. et al. Identificação de Cooperia punctata (Linstow, 1907) resistente a ivermectin e doramectin em bovinos no estado do Rio de Janeiro, Brasil. In: CONGRESSO BRASILEIRO DE PARASITOLOGIA VETERINÁRIA, 12., 2002, Rio de Janeiro. Anais... Rio de Janeiro, 2002.

COLES, G.C.; BAUER, C.; BORGSTEEDE, F.H.M. et al. World Association for the Advancement of Veterinary Parasitology (WAAVP) methods for detection of anthelmintic resistance in nematodes of veterinry importance. Vet. Parasitol., v.44, p.35-44, 1992.

ECHEVARRIA, F. Resistência a antihelmínticos. In: CONGRESSO BRASILEIRO DE PARASITOLOGIA VETERINÁRIA, 12 ., 2002, Rio de Janeiro. Anais... Rio de Janeiro, 2002. 


\section{Rangel et al.}

ECHEVARRIA, F.; BORBA, M.F.S.; PINHEIRO, A.C.; et al. The prevalence of anthelmintic resistance in nematodes parasites of sheep in Southern Latin America: Brazil. Vet. Parasitol., v.62, p.199-206, 1996.

FIEL, C.A.; SAUMELL, C.A.; STEFFAN, P.E. et al. Resistance of Cooperia to ivermectin treatments in grazing cattle of the Humid Pampa, Argentina. Vet. Parasitol., v.97, p.211-217, 2001.

GORDON, H.M.; WHITLOCK, H.V.A., New technique for counting nematodes eggs in sheep faeces. J. Coun. Sci. Ind. Res. Aust., v.12, p.50$52,1939$.

MOTTIER, L; LANUSSE, C. Bases moleculares de la resistência fármacos anti-helmínticos. Rev. Med. Vet., v.82, p.74-85, 2001.

ROBERTS, F.; O'SULLIVAN, P. Methods for eggs counts and larvae cultures for strongyles infesting gastrointestinal tract of cattle. Aust. J. Agric. Res., v.1, p.99-102, 1949.
SOUZA, A.P.; RAMOS, C.I.; DALAGNOL, C. et al. Resistência de helmintos gastrintestinais de bovinos a anti-helmínticos no estado de Santa Catarina. In: CONGRESSO BRASILEIRO DE PARASITOLOGIA VETERINÁRIA, 12., 2002, Rio de Janeiro. Anais... Rio de Janeiro, 2002.

TAYLOR, M.A.; HUNT K.R.; GOODYEAR K.L. Anthelmintic resistance detection methods. Vet. Parasitol., v.103, p.183-194, 2002.

TORRANO, C. Mode of action and resistance mechanisms of moxidectin and macrocyclic lactones. In: INTERNATIONAL SEMINAR IN ANIMAL PARASITOLOGY, 5., 2003, Merida. Anais... Merida, 2003.

VERÍSSIMO, C.J.; OLIVEIRA S.M.; SPÓSITO FILHA, E. Eficácia de alguns anti-helmínticos em uma ovinocultura no estado de São Paulo, Brasil. In: CONGRESSO BRASILEIRO DE PARASITOLOGIA VETERINÁRIA, 12., 2002, Rio de Janeiro. Anais... Rio de Janeiro, 2002. 